Int. J. Dev. Biol. 48: 731-738 (2004)

doi: $10.1387 / \mathrm{ijdb} .041874$ th

\title{
Blind cavefish and heat shock protein chaperones: a novel role for hsp90 $\alpha$ in lens apoptosis
}

\author{
THOMAS A. HOOVEN, YOSHIYUKI YAMAMOTO and WILLIAM R. JEFFERY* \\ Department of Biology, University of Maryland, College Park, MD, USA
}

\begin{abstract}
Lens apoptosis plays a central role in cavefish eye degeneration. Heat shock proteins (hsps) can regulate apoptosis; therefore, we examined the relationship between constitutive hsp70 and hsp90 expression and lens apoptosis. The model system is Astyanax mexicanus, a teleost species consisting of an eyed surface-dwelling (surface fish) form and numerous blind cavedwelling (cavefish) forms. Optic primordia are formed in the cavefish embryo but they subsequently undergo lens apoptosis, arrest in development and degenerate. Astyanax hsp90 and hsp70 DNAs were isolated to use as probes to compare gene expression during surface fish and cavefish development. Hsp90 $\beta$, which encodes one of two hsp90 isoforms, was not expressed in the surface fish or cavefish lens, whereas hsp70was expressed in the lens of both forms, suggesting that neither is directly involved in lens apoptosis. In contrast, $h s p 90 \alpha$, the other hsp90 isoform, was expressed in the cavefish but not the surface fish lens. Hsp90 $\alpha$ expression peaked shortly before the beginning of lens apoptosis in three convergent cavefish populations, suggesting a close relationship with lens apoptosis. The absence of hsp90 $\beta$ in the lens allowed us to use geldanamycin and radicicol, specific inhibitors of hsp90 chaperone function, to determine whether lens cell death requires hsp90 $\alpha$ expression. Both inhibitors blocked TUNEL labeling in the cavefish lens, suggesting that $h s p 90 \alpha$ is required for apoptosis. In contrast to their effects on the lens, these inhibitors induced TUNEL labeling in the surface epidermis, presumably due to effects on hsp90 $\beta$ function, implying that the two-hsp90 isoforms may have contrasting roles in cell survival. We conclude that hsp90 $\alpha$ plays a novel role in lens apoptosis and cavefish eye degeneration.
\end{abstract}

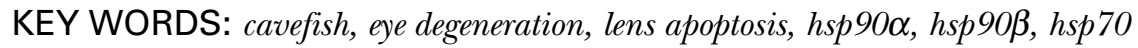

\section{Introduction}

The heat shock proteins (hsps) are members of a small and highly conserved gene family encoding molecular chaperones: the cytoplasmic mediators of protein folding and metabolism. Three major hsp subgroups are distinguished by their relative molecular masses: hsp90 ( 90kDa), hsp70 ( 70kDa), and the low molecular weight hsps (16-47kDa). Although first identified as stress-inducible proteins, hsps were subsequently shown to have critical roles in basic cellular and developmental processes (Morimoto etal.,1994; Richter and Buchner, 2001). Hsp70 is generally required for correct folding, assembly, intracellular targeting, and degradation of polypeptides and oligomeric proteins (Morimoto et al., 1994). Under normal environmental conditions, hsp70 is strongly expressed in the lens of zebrafish (Blechinger et al., 2002) and chick (Dash et al., 1994) embryos. Hsp90 has more specialized chaperoning roles and most of its client proteins are members of key signal transduction networks (Young et al., 2001), such as the steroid hormone receptors (Picard et al., 1990) and signaling kinases (Xu and Lindquist, 1993). Two hsp90 isoforms, hsp90 $\alpha$ and hsp90 $\beta$, are encoded by different genes and exhibit distinct developmental expression patterns in teleosts (Krone and Sass, 1994) and mammals (Gupta, 1995). In zebrafish, hsp90 $\alpha$ is involved in somite and axial muscle development (Lele et al., 1999), whereas hsp90 $\beta$ is preferentially expressed in the anterior epidermis and central nervous system (CNS), where its role is unknown (Krone and Sass, 1994).

Hsps are also implicated in evolutionary processes. Hsp70 can facilitate mutations through its involvement in a number of DNA sequence-alteration activities (Ogata et al., 1993; Chow, 2000). Hsp90 is thought to capacitate phenotypic variation and morpho-

Abbreviations used in this paper: hsp, heat shock protein.

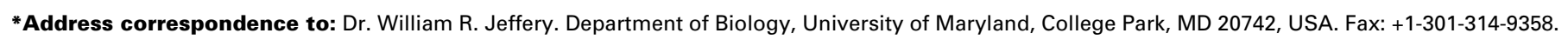
e-mail: jeffery@umd.edu

GenBank Accession Number: Hsp90 $\alpha$ cDNA clone was deposited in GenBank under Accession Number AY222612 

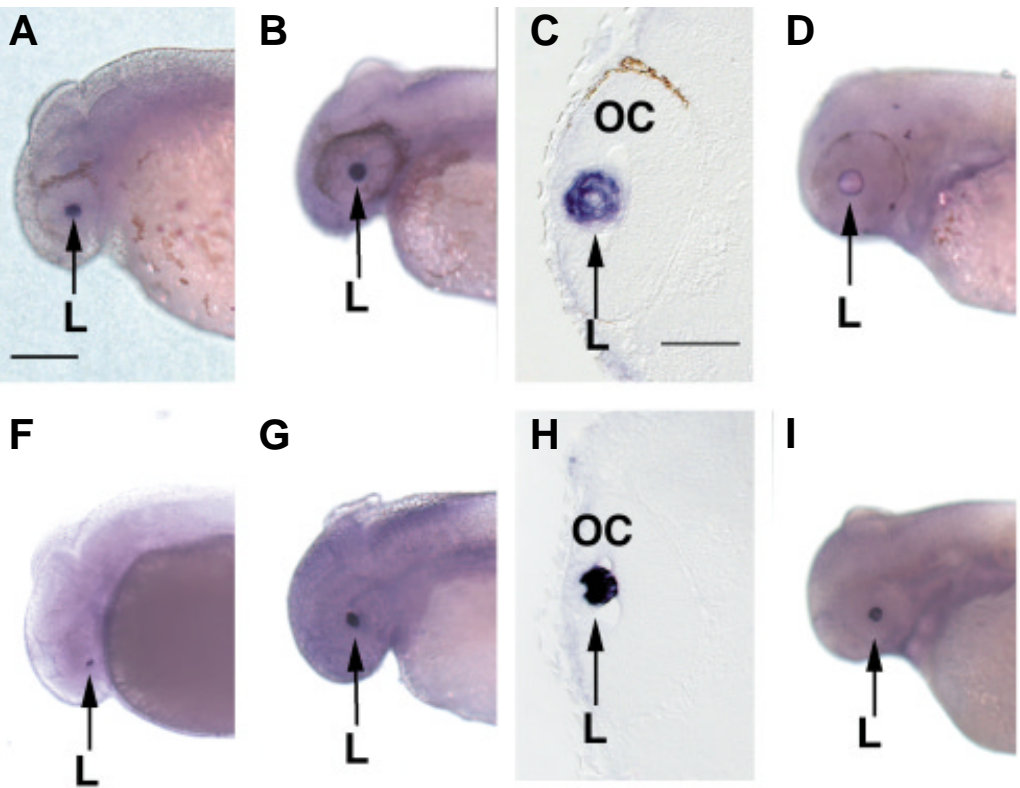

G
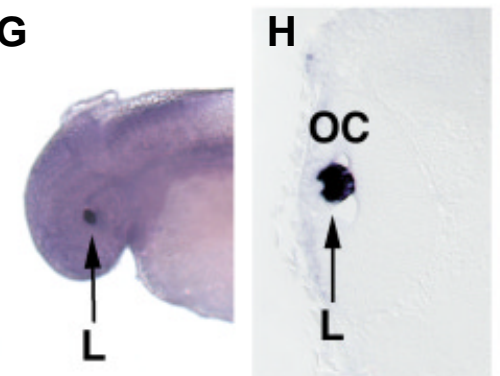

I

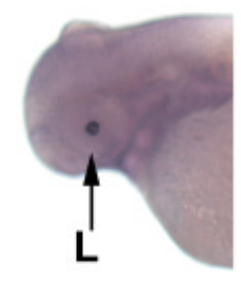

Fig. 1. Non-stress related hsp70 expression in the lens during surface fish (A-E) and Pachón cavefish (F- J) development. Whole mount in situ hybridizations of 24 (A, F), 36 (B, G), and 48 (D, I) hpf embryos with developing optic regions viewed from a lateral side. Sections through in situ hybridized embryos showing the optic primordia at $36(C, H)$ and $48(E, J)$ hpf. L: lens. OC: optic cup. LE: lens epithelium. Scale bar in $A$ is $100 \mu \mathrm{m}$; magnification is the same in $A, B, D, F, G, I$. Scale bar in $C$ is $50 \mu \mathrm{m}$; magnification is the same in $C, E, H, J$.

logical evolution during periods of environmental flux by chaperoning developmental signaling components and masking phenotypic variation under normal environmental conditions (Rutherford and Lindquist, 1998; Queitsch et al., 2002; Sollars et al., 2003). Further understanding of the multiple roles of hsp proteins in development should contribute to our knowledge of how evolutionary diversity is generated.

We study the evolution of development in the teleost Astyanax mexicanus, a single species consisting of an eyed surface-dwelling form (surface fish) and several blind cave-dwelling (cavefish) forms (Jeffery, 2001). There are about 30 different populations of A. mexicanus cavefish with different genetic backgrounds and converged eyeless phenotypes (Dowling et al., 2002). Although adult cavefish have degenerate eyes, eye primordia begin to develop in embryos but they later arrest and degenerate. The first point of divergence between surface fish and cavefish eye development occurs during lens differentiation. The fiber cells, which normally elongate and begin to express crystallin proteins, do not terminally differentiate and instead undergo apoptosis in the cavefish lens (Jeffery and Martasian, 1998; Yamamoto and Jeffery, 2000). In addition, the iris, cornea, and anterior chamber do not appear during cavefish eye development. The retina, which initially forms properly, does not differentiate the normal number of photoreceptor cells and gradually stops growing, although new retinal cells continue to be produced in the ciliary marginal zone (Strickler et al., 2002).

The embryonic lens is an organizer of eye development (Beebe, 2000; Yamamoto and Jeffery, 2000; Kurita et al., 2003). In blind cavefish, loss of this organizer activity through lens apoptosis results in eye degeneration. The central role of the lens in this process was demonstrated by lens transplantation experiments (Yamamoto and

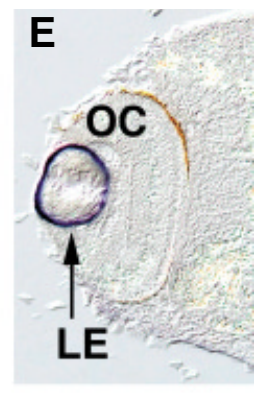

$\mathbf{J}$

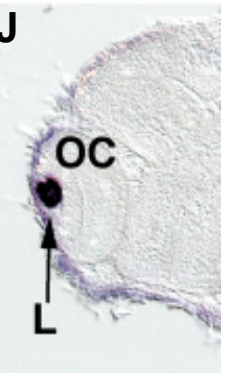

Jeffery, 2000; Jeffery et al., 2003). When a cavefish lens vesicle was placed into a surface fish optic cup lacking its own lens vesicle it underwent apoptosis and degeneration on schedule. Similar to the degenerate cavefish eye, the surface fish eye with the transplanted lens did not develop an iris, cornea, or anterior chamber, and retinal growth was inhibited. In the reciprocal experiment, a surface fish lens vesicle was transplanted into a cavefish optic cup after its own lens vesicle was removed. The transplanted surface fish lens developed normally in the cavefish host: an iris, cornea, and anterior chamber were formed, the retina increased in size, and photoreceptor cells differentiated. These results indicate that (1) apoptosis is an autonomous property of the cavefish lens, (2) the surface fish lens is capable of inducing the cavefish eye primordium to resist degeneration and develop normally, and (3) the cavefish lens has lost this inductive ability, presumably because of apoptosis. The cavefish system opens new opportunities to study the role of apoptosis in generating an evolutionary change in development.

In addition to their other roles, hsps are also involved in apoptosis (Xanthoudakis and Nicholson, 2000). Hsp70 has been shown to protect cells from stress-induced apoptosis (Mosser et al., 2000) by binding to the cell death mediator procaspase- 9 and preventing its recruitment to the Apaf-1 apoptosome (Beere et al., 2000). There is also evidence that hsp90 is involved in apoptosis (Pandy et al., 2000; Wu et al., 2002; Vanden Berghe et al., 2003), although the mechanisms are still unclear. Because hsps play important roles in apoptosis, we have studied the expression patterns of $h s p 70, h s p 90 \alpha$, and $h s p 90 \beta$ during cavefish lens development.

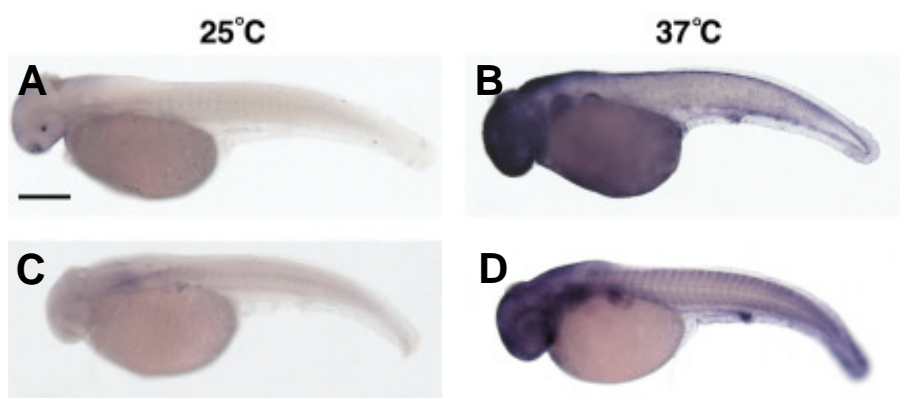

Fig. 2. Non-stress $(A, C)$ and heat shock induced $(B, D)$ patterns of hsp70 (A,B) and hsp90 $(\mathrm{C}, \mathrm{D})$ expression in 36 hpf Pachón cavefish embryos. Whole mount in situ hybridizations were developed for the same time in controls and heat shocked embryos. Hsp90 $\alpha$ is not seen in the lens or myostomes in $(C)$ because the reaction was stopped early in order to avoid excess stain development after heat shock in (D). Scale bar in $(A)$ is $200 \mu \mathrm{m}$; magnification is the same in all frames. 


\section{Chica}

A

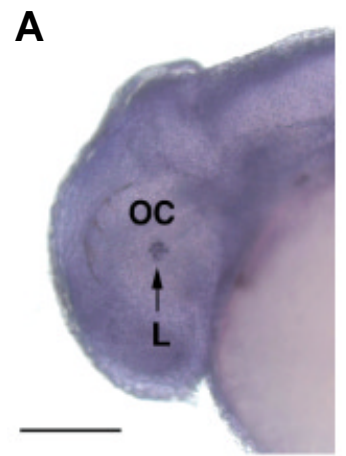

C

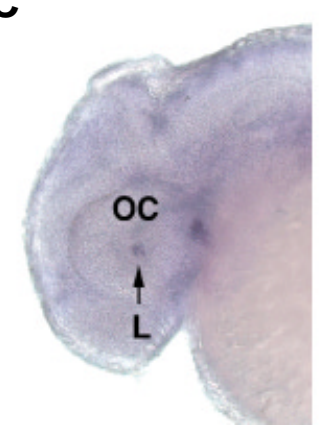

Tinaja

B

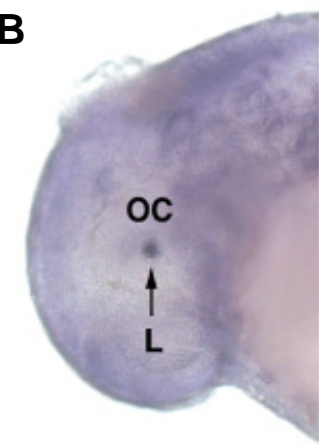

D

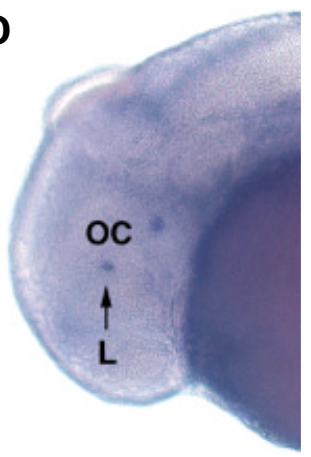

Fig. 3. Non-stress related $h s p 70$ (A,B) and $h s p 90 \alpha(C, D)$ expression in the lens of $\mathbf{3 6} \mathrm{hpf}$ Chica (A,C) and Tinaja (B,D) cavefish embryos. Labels are the same as in Fig. 1. Scale bar in (A) is $100 \mu \mathrm{m}$; magnification is the same in all frames.

We found that the patterns of $h s p 70$ and $h s p 90 \beta$ expression in surface fish and cavefish embryos are essentially the same and do not suggest a direct role in lens apoptosis. However, we demonstrate here that $h s p 90 \alpha$ is specifically activated prior to lens apoptosis in several different cavefish populations and that pharmacological inhibition shows that $h s p 90 \alpha$ activation is necessary for inducing apoptosis in the cavefish lens. Our results suggest a novel role for hsp90a in lens apoptosis and eye degeneration in the blind cavefish.

\section{Results}

\section{Hsp70 is expressed in the cavefish lens}

To determine whether hsp70 downregulation prcedes lens apoptosis in cavefish, we cloned an Astyanax hsp70DNA fragment to generate an in situhybridization probe. The hsp70DNA showed $86 \%$ and $95 \%$ conservation at the nucleotide level and amino acid levels respectively to zebrafish $h s p 70$ (Lele et al., 1997).

In situhybridization was conducted on surface fish and Pachón cavefish embryos between 16 and $72 \mathrm{hpf}$ (Fig. 1). At normal growth temperature $\left(25^{\circ} \mathrm{C}\right)$, hsp70 expression was restricted to the developing lens (Figs. 1,2A), as described previously in zebrafish (Blechinger et al., 2002). The pattern of $h s p 70$ expression was distinct from that of $h s p 90 \alpha$ and $h s p 90 \beta$ (see Figs. 4,5). The constitutive hsp70 mRNA level was greatly increased by a $1 \mathrm{hr}$ $37^{\circ} \mathrm{C}$ heat shock and expression was expanded throughout surface fish and cavefish embryos (Fig. 2 A,B; data not shown), indicating that $h s p 70$ is heat inducible. In surface fish and cavefish

developing at normal temperature, hsp70mRNA was first detected at $24 \mathrm{hpf}$, shortly after the lens vesicle was formed from surface ectoderm (Fig. 1 A,F). Hsp70mRNA continued to be present in the surface fish and cavefish lens through $72 \mathrm{hpf}$ (Fig. $1 \mathrm{~B}-\mathrm{E}, \mathrm{G}-\mathrm{J}$; data not shown), when lens apoptosis is occurring in cavefish (Jeffery and Martasian, 1998; Strickler and Jeffery, unpublished). In surface fish, hsp70 expression was attenuated to the lens epithelium beginning between 36 and 48 hpf (Fig. 1 D,E), as crystallincontaining fiber cells begin to differentiate in the central core of the lens. At this time, hsp70 mRNA was still present throughout the apoptotic cavefish lens (Fig. $1 \mathrm{I}, \mathrm{J}$ ), which does not produce elongated lens fiber cells, although it does accumulate crystallin family mRNA and protein (Jeffery et al., 2000). The latter was the only difference observed in hsp70 expression during surface fish
A
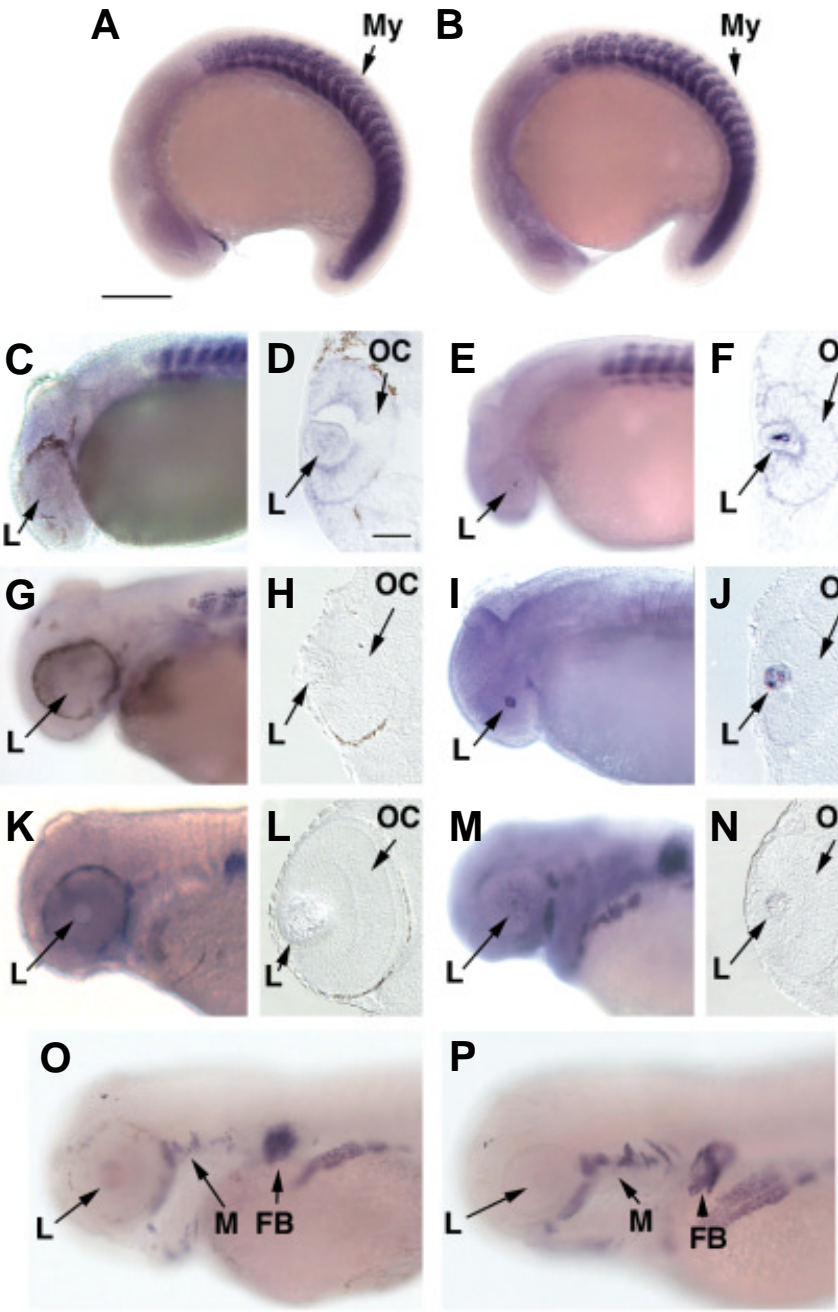
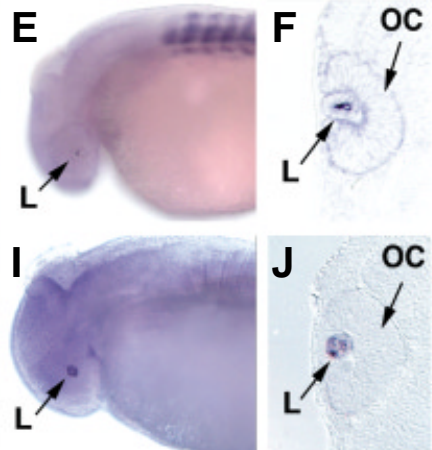

Fig. 4. Non-stress related $h s p 90 \alpha$ expression during surface fish (A, C, D, G, H, K, L, O) and Pachón cavefish (B, E, F, I, J, M, N, P) development. Whole mount in situ hybridizations of 18 somite $(A, B), 24 \mathrm{hpf}(C, E), 36 \mathrm{hpf}$ $(G, I), 48 \mathrm{hpf}(K, \mathrm{M})$, and $72 \mathrm{hpf}(\mathrm{O}, \mathrm{P})$ embryos with developing optic regions viewed from a lateral side. Sections through the optic primordia of whole mount in situ hybridizations at $24(D, F), 36(H, J)$ and $48(L, N)$ hpf. My, myotome; $F B$, fin bud; $M$, eye and pharyngeal muscles. Other labels are the same as in Fig. 1. Scale bar in (A) is $100 \mu \mathrm{m}$; magnification is the same in $(A-C, E, G, I, K, M, O, P)$. Scale bar in (D) is $30 \mu \mathrm{m}$; magnification is the same in $(D, F, H, J, L, N)$. 
A

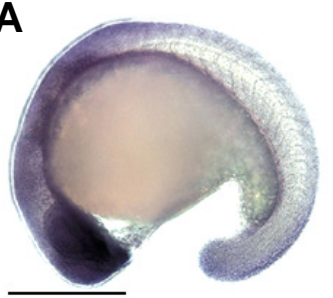

SE
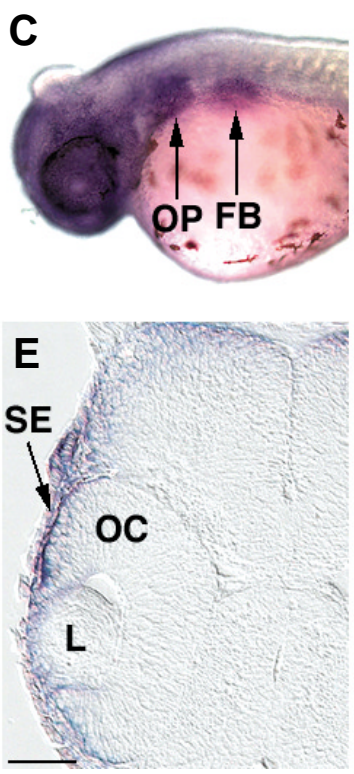

B
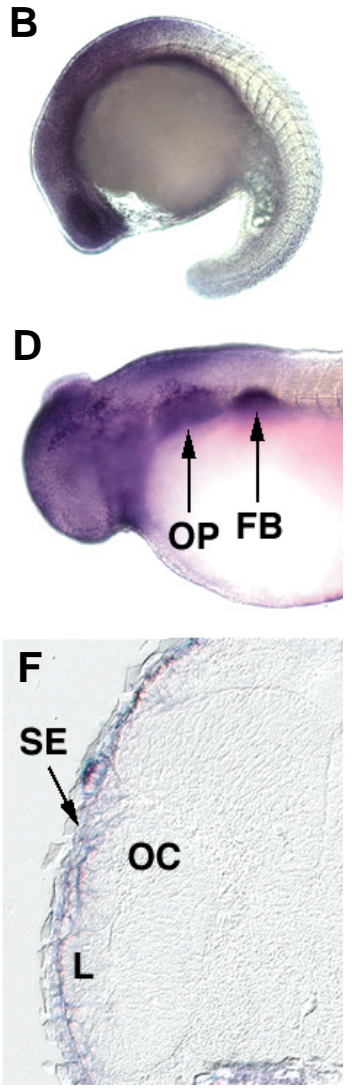

Fig. 5. Non-stress related $h s p 90 \beta$ expression during surface fish (A, C, E) and Pachón (B, D, F) cavefish development. Whole mount in situ hybridization of 18 somite $(A, B)$ and $36 \mathrm{hpf}(C, D)$ embryos with developing optic regions viewed from a lateral side. (E,F) Sections through the optic primordia of whole mount in situ hybridizations at 36 hpf. OP, otic placode. Other labels are the same as in Figs. 1,4. Scale bar in (A) is $100 \mu \mathrm{m}$; magnification is the same in (A-D). Scale bar in (E) is $50 \mu \mathrm{m}$; magnification is the same in $(D, E)$.

and cavefish lens development. The results show that $h s p 70$ expression is stable during lens apoptosis in Pachón cavefish.

Other cavefish populations also exhibit lens apoptosis as a prelude to eye degeneration (Jeffery et al., 2003). To assess whether hsp70 is involved in lens apoptosis in some of these populations, the $h s p 70$ expression analysis was extended to Chica and Tinaja cavefish embryos. The results showed that $h s p 70$ mRNA was also expressed during the period of lens apoptosis in these divergent cavefish populations (Fig. 3 A,B). Therefore, we conclude that $h s p 70$ expression is not downregulated during cavefish lens development and that hsp70 may not be directly involved in lens apoptosis.

\section{Hsp90 $\alpha$ expression is activated in the cavefish lens}

To determine if other hsps may be involved in lens apoptosis, we compared $h s p 90 \alpha$ and $h s p 90 \beta$ expression in surface fish and Pachón cavefish embryos. A full-length $h s p 90 \alpha$ Astyanax cDNA and an $h s p 90 \beta$ DNA fragment were isolated. The $h s p 90 \alpha$ cDNA sequence showed $84 \%$ identity at the nucleotide level and $94 \%$ identity at the amino acid level to zebrafish hsp900 (Krone and Sass, 1994). The $h s p 90 \beta$ DNA was $84 \%$ identical at the nucleotide

level and $95 \%$ identical at the amino acid level to zebrafish $h s p 90 \beta$ (Krone and Sass, 1994).

In situhybridizations were carried out between 16 and $72 \mathrm{hpf}$ in surface fish and Pachón cavefish embryos (Fig. 4). Similar to the expression pattern seen in zebrafish embryos (Sass et al., 1996; 1999), hsp900 mRNA was restricted to developing myotomes in early Astyanax embryos developing at 25ㅇ (Fig. 4 A, B, C, E, G). Later, between 36 and $48 \mathrm{hpf}$, hsp90a mRNA decreased in the myotomes and was seen in developing muscle around the eye, pharyngeal muscle, pectoral fin buds, and a strip of tissue above the yolk mass that may represent developing gut muscle (Fig. $4 \mathrm{~K}$, $\mathrm{M}, \mathrm{O}, \mathrm{P})$. Expression in these tissues was the same in surface fish and cavefish embryos. A $37^{\circ} \mathrm{C}$ heat shock resulted in the intensification and expansion of $h s p 90 \alpha$ expression (Fig. 2 C,D), indicating that $h s p 90 \alpha$ is heat inducible in both forms of Astyanax. Remarkably, beginning at $24 \mathrm{hpf}, h s p 900$ expression was detected in the cavefish lens vesicle (Fig. $4 \mathrm{E}, \mathrm{F}, \mathrm{I}, \mathrm{J}$ ). Hsp900 expression in the cavefish lens peaked at $36 \mathrm{hpf}$ and disappeared after $48 \mathrm{hpf}$ (Fig. $4 \mathrm{I}, \mathrm{J}, \mathrm{M}, \mathrm{N}$ ). The temporal pattern of $h s p 90 \alpha$ expression suggests that it presages lens apoptosis, which according to TUNEL analysis peaks at $48 \mathrm{hpf}$ (Jeffery and Martasian, 1998; Strickler and Jeffery, unpublished). Hsp90a mRNA was not detectable during surface fish lens development (Fig. 4 C, D, G, H, K, L, O). As in zebrafish (Lele et al., 1999), the pattern of $h s p 90 \beta$ expression differed entirely from that of $H s p 90 \alpha$. In situ hybridization showed $h s p 90 \beta$ transcripts in the anterior epidermis and CNS, otic placodes, and pectoral fin buds, but not in the lens, and there were no differences in surface fish and cavefish (Fig. 5). The results show that $h s p 90 \alpha$, but not $h s p 90 \beta$, is specifically activated prior to the beginning of apoptosis in the Pachón cavefish lens.

When in situ hybridization was extended to other cavefish populations, we found that $h s p 90 \alpha$ expression also peaked before apoptosis in the lens vesicles of Chica and Tinaja cavefish embryos (Fig. $3 \mathrm{C}, \mathrm{D}$ ). The results suggest that Hsp900 may be involved in lens apoptosis in diverse cavefish populations.

\section{Hsp90 $\alpha$ inhibition shows a requirement for lens apoptosis}

Hsp900 activation in the cavefish lens could be the cause or effect of apoptosis. We initially attempted to study the role of $h s p 90 \alpha$ in lens apoptosis by injecting antisense $h s p 90$ a morpholinos into cavefish eggs and $h s p 90 \alpha$ mRNA into surface fish eggs. However, morpholino injection at several different concentrations resulted in embryonic lethality prior to the formation of a morphologically distinguishable eye primordium, probably because of the major role of this molecular chaperone in early somite development (Lele et al., 1999). Furthermore, injection of hsp90a mRNA produced no recognizable phenotype, perhaps due to the difficulty of

\section{TABLE 1}

\section{SUPPRESSION OF LENS APOPTOSIS IN CAVEFISH EMBRYOS BY} HSP9O INHIBITION

\begin{tabular}{|c|c|c|c|c|c|c|}
\hline \multirow[t]{2}{*}{ Treatment } & \multicolumn{5}{|c|}{ Number of lenses } & \multirow[t]{2}{*}{ Total lenses $(\mathrm{N})$} \\
\hline & $\begin{array}{l}\text { Number of TUNEL } \\
\text { stained nuclei in section }\end{array}$ & 0 & $1-5$ & $6-10$ & $10+$ & \\
\hline Control & & 0 & 1 & 3 & 10 & 14 \\
\hline Geldanamycin & & 5 & 11 & 7 & 2 & 25 \\
\hline Radicicol & & 4 & 6 & 4 & 0 & 14 \\
\hline
\end{tabular}



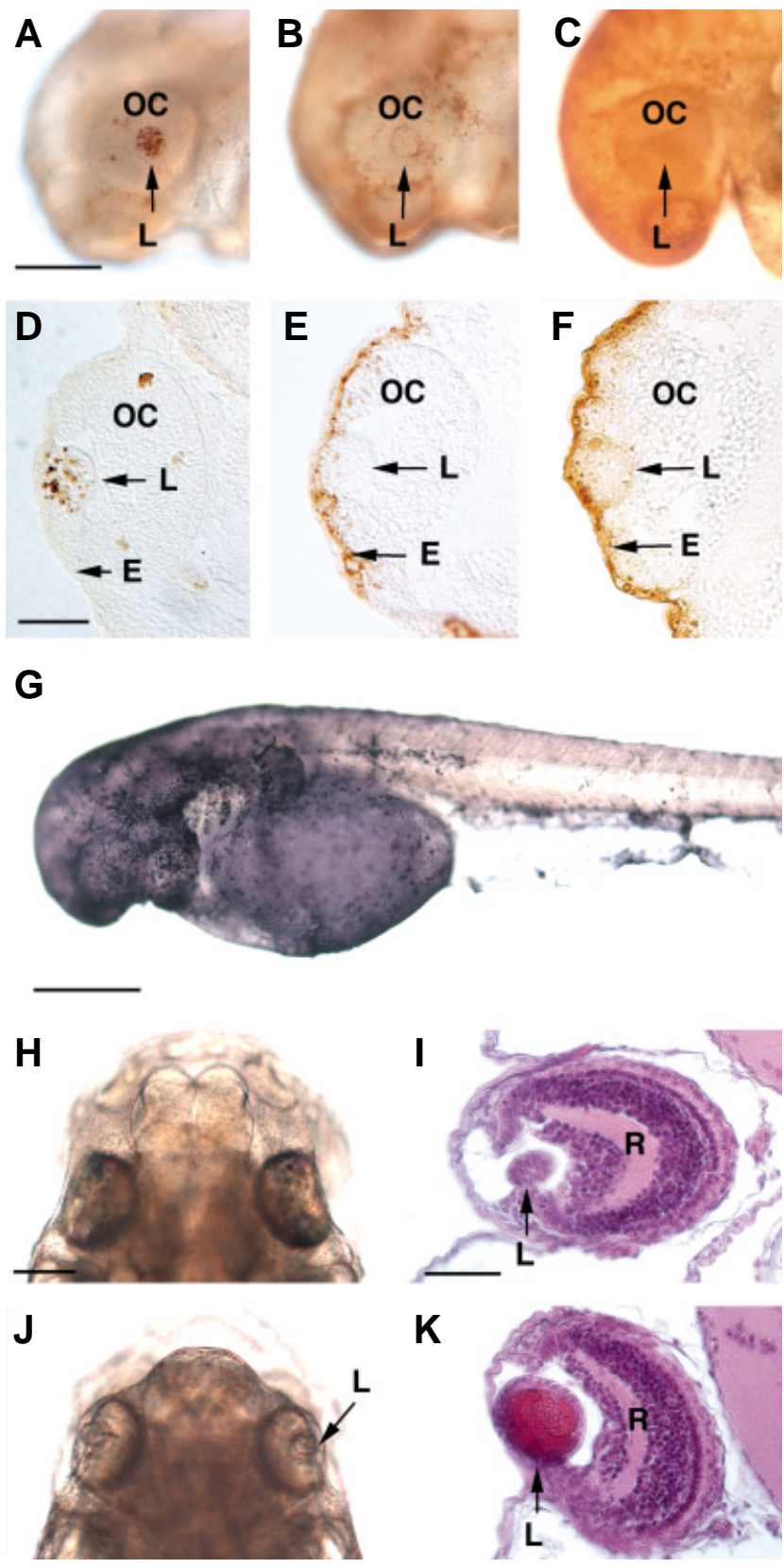

partitioning mRNA to the lens in sufficient concentration to elicit an effect. We next examined the effects of geldanamycin and radicicol on lens apoptosis. These structurally different compounds specifically inhibit hsp90 chaperone function by preventing the ATPdependent release of client proteins (Whitesell et al., 1994; Roe et al., 1999).

Cavefish embryos were pre-treated with geldanamycin or radicicol beginning at $24 \mathrm{hpf}$ and the drug treated and control embryos were fixed and subjected to TUNEL assay at $48 \mathrm{hpf}$, during the peak of lens apoptosis. The results showed that lens apoptosis was greatly reduced relative to controls when embryos were treated with either drug (Fig. 6 A-F; Table 1). In drug-treated embryos, however, hsp900 expression still peaked at $36 \mathrm{hpf}$ (data not shown), indicating that the pharmacological effects cannot be attributed to a delay in the normal program of cavefish lens
Fig. 6. Effects of pharmacological inhibition of hsp90 on cavefish lens apoptosis. (A-F) TUNEL stained whole mounts showing lateral views of the eye (A-C) and corresponding sections through the lens and optic cup (D-F) in control $(A, D), 20 \mu \mathrm{g}$ geldanamycin-treated $(B, E)$, and radicicoltreated (C,F) cavefish embryos at $48 \mathrm{hpf}$. After drug treatment, apoptotic cells were less prevalent in the lens but appeared in the surface ectoderm (E,F). (G) A 56 hpf cavefish embryo treated with $10 \mu \mathrm{g} / \mathrm{m} /$ geldanamycin showing extensive TUNEL labeling in anterior epidermis. (H-K) Rescue of lens growth and differentiation in cavefish treated with radicicol. $(\mathbf{H}, \mathbf{I})$ Controls. (J,K) Radicicol treatment. $(\mathrm{H}, \mathrm{J})$ Dorsal views of the optic regions at $6 \mathrm{dpf}$ showing increased lens size after radicicol treatment (K). (I,K) Sections through the lens and optic cup showing eosin-stained central core indicative of lens fiber differentiation after radicicol treatment (K). Controls do not show lens core development (I). E, epidermis. R, Retina. Other labels are the same as in Fig. 1. Scale bar in (A) is $100 \mu \mathrm{m}$; magnification is the same in (A-C). Scale bar in (D) is $50 \mu \mathrm{m}$. Scale bar in (G) is $200 \mu \mathrm{m}$; magnification is the same in $(G, I)$. Scale bar in $(H)$ is $60 \mu \mathrm{m}$; magnification is the same in $(\mathrm{H}, \mathrm{J})$. Scale bar in (I) is $50 \mu \mathrm{m}$; magnification is the same in $(I, \mathrm{~K})$.

development. Subsequent development was followed in some of the radicicol treated embryos (Fig. $6 \mathrm{H}-\mathrm{K}$ ). At 6 days post-fertilization (dpf), the size of the lens was markedly increased relative to controls and fiber cell differentiation was rescued in some of these embryos, as determined by the formation of a crystalline lens core (Fig. 6K), a feature not seen in untreated controls (Fig. 6I). The results indicate that inhibition of hsp90 function can prevent lens apoptosis, suggesting that it has a causal role in this process.

In striking contrast to their inhibitory effects on lens apoptosis, geldanamycin and radicicol induced apoptosis in the surface epidermis (Fig. 6 E-G). Since these drugs inhibit both hsp90 $\alpha$ and hsp90 $\beta$ function (Lele et al., 1999), and the latter is expressed in surface epidermis (Fig. 5), we conclude that hsp90 $\beta$ normally protects these cells from apoptosis during early development. The results suggest that different hsp90 isoforms may have contrasting roles in promoting or suppressing apoptosis.

\section{Discussion}

We have studied $h s p 70$ and hsp90 expression during lens apoptosis in the cavefish Astyanax. Our results suggest that $h s p 70$ and $h s p 90 \beta$ are not directly responsible for lens apoptosis. However, we demonstrate that $h s p 90 \alpha$, which is expressed in the cavefish lens immediately prior to the beginning of apoptosis, is required for lens cell death. Our studies suggest a novel role for the molecular chaperone hsp $90 \alpha$ in the evolution of lens apoptosis and the eyeless phenotype in blind cavefish.

The incentive for considering hsp70 as a regulator of cavefish lens apoptosis was based on two previous findings. First, under non-stress conditions, hsp70 is known to be expressed in the lens during early chick and zebrafish development (Dash et al., 1996; Blechinger et al., 2000). The role of hsp70 expression in the developing lens is unknown. Second, hsp70 has been shown to act as a survival factor, protecting mammalian cells from stressinduced apoptosis by direct physical interaction with components of the cell death machinery (Beere et al., 2000). Thus, we hypothesized that hsp70 may protect developing lens cells from apoptosis during the critical steps of lens morphogenesis and differentiation and that hsp70 loss could trigger apoptosis in the cavefish lens. As in zebrafish (Blechinger et al., 2002), we found that hsp70 is 
strongly expressed in the developing Astyanaxlens. There are no striking changes in hsp70 expression, however, either between surface fish and cavefish or in the developing cavefish lens before or during apoptosis. Three different cavefish populations failed to show changes in hsp70 expression associated with lens apoptosis. Thus, blind cavefish do not appear to activate the lens cell death pathway by down-regulating hsp70. Our results suggest that hsp70 is not directly involved in cavefish lens apoptosis, although they do not discount the possibility that this molecular chaperone may function as a survival factor during lens development. If hsp70 does have a survival role in the lens our results imply that it must somehow be negated by the activity of hsp $90 \alpha$.

The analysis of hsp90 expression during Astyanax development was instigated by mounting evidence that hsp90, which has a much more specific range of chaperone activities and client proteins than hsp70, may be a regulator of apoptosis (Xanthoudakis and Nicholson, 2000). We report here that hsp90 $\alpha$ is activated in the cavefish lens during embryonic development. The lens is the only tissue, other than developing somites and muscle cells, where we observed reproducible $h s p 90 \alpha$ expression during early cavefish development. Moreover hsp900 is the first gene we have discovered that is exclusively expressed in the cavefish lens. Other lens specific genes, such as those encoding $\beta$ - and gamma crystallins, are expressed in both surface fish and cavefish embryos (Jeffery et al., 2000). Expression of hsp90ß, the other hsp90 isoform, could not be detected in the Astyanaxlens, although it was expressed the anterior epidermis, CNS, and otic placodes, as previously reported in zebrafish (Krone and Sass, 1994; Lele et al., 1999). Our results show that the two hsp90 isoforms have distinct expression patterns during Astyanax surface fish and cavefish development.

Several lines of evidence support the hypothesis that hsp900 activation is responsible for apoptosis in the cavefish lens. First, hsp900 expression peaks during a narrow window of time immediately prior to the beginning of nuclear DNA fragmentation as detected by TUNEL in dying cavefish lens cells. Second, hsp900 expression occurs during this critical time interval in three divergent cavefish populations (Dowling et al., 2002). This evolutionary convergence in $h s p 90 \alpha$ activation suggests a close relationship with eye degeneration. Third, inhibition of hsp90 function by geldanamycin or radicicol can block lens apoptosis. Although the two drugs interfere with the chaperone activity of both hsp90 isoforms (Lele et al., 1999), the absence of hsp90 $\beta$ expression in the lens points to hsp90 $\alpha$ as the critical apoptotic regulator. These studies rule out the possibility that hsp $90 \alpha$, acting as a survival factor, is expressed in the cavefish lens in a final but unsuccessful bid to prevent cell death. If this were the case, apoptosis would not be expected to decrease following hsp90 inhibition.

Most previous work has implicated hsp90 as a protector rather than an inducer of apoptosis. For example, stabilization of RIP-1 kinase, an anti-apoptotic protein, is dependent on binding with hsp90 (Lewis et al., 2000; Vanden Berghe et al., 2003). In the absence of hsp90, RIP-1 is degraded and cells are sensitized to apoptosis. The only other indication of hsp $90 \alpha$ pro-apoptotic activity is a recent report suggesting that it promotes nicotine-induced apoptosis in human cultured cells (Wu et al., 2002). The present results implicating hsp90 $\alpha$ in lens apoptosis suggest that this molecular chaperone may be able to promote or prevent apoptosis in different tissues and at different times in development, perhaps depending on the client protein(s) being chaperoned. It is also clear that in some situations hsp90 $\alpha$ is neutral with regard to apoptosis. A case in point is that although $h s p 90 \alpha$ is strongly expressed in teleost myotomes and other muscle cells (the present study; Sass et al., 1996), these tissues do not show extensive apoptosis during Astyanax development (Hooven and Jeffery, unpublished).

It is conceivable that hsp $90 \alpha$ and hsp $90 \beta$ have contrasting roles in cell death: the former promoting and the latter preventing apoptosis. This possibility is supported by our results showing geldanamycin and radicicol inhibition of apoptosis in the lens, where $h s p 90 \alpha$ is expressed, but induction of apoptosis in the surface epidermis, where hsp90ß is expressed. Interestingly, most previous pharmacological and biochemical studies, including those implicating hsp90 as a survival factor, have not distinguished between the individual effects of its two isoforms.

It remains unclear as to exactly how hsp $90 \alpha$ may initiate apoptosis in the cavefish lens. Two alternative models are proposed here, assuming that the hsps in question vary in the same fashion as their mRNAs. The first possibility is that hsp90 $\alpha$ may initiate lens apoptosis directly through binding to and activating a component of the cell death machinery, such as pro-caspase 3. In this light, it must be considered that lens fiber cells undergo a unique process resembling apoptosis during normal differentiation, which is required for the elimination of organelles and optical clarification (Lang, 1997; Wride, 2000). Accordingly, bcl-2 and caspase family proteins are normally expressed in the chick lens, and $b c /-2$ overexpression and caspase inhibitors can block fiber cell differentiation (Wride et al., 1999). Therefore, hsp90 $\alpha$ activation could function somewhere in the cell death pathway downstream of caspase-3 to drive the lens apoptotic process to completion. Further studies of the factors activated downstream of hsp $90 \alpha$ could provide insight into how it promotes apoptosis.

The alternative possibility is that $h s p 90 \alpha$ mediates lens apoptosis indirectly by interfering with the normal protective function of an anti-apoptotic factor or hsp. Hsp70, which suppresses caspase activation by targeting Apaf- 1 and the anti-apoptoic protein Bag-1, would be a prime candidate for this interaction, although there are others, including hsp27, which also has a major role in cell survival (Xanthoudakis and Nicholson, 2000). During activation of the estrogen receptor complex, hsp70 binding is followed by an interaction with dimeric hsp90 (Smith et al., 1992; Chen and Smith, 1998; Hutchison et al., 1994), an indication that multimeric protein complexes containing both hsp70 and hsp90 are present in some cells. In order to distinguish between the direct and indirect models for hsp90 $\alpha$ function, it will be necessary to investigate hsp90 $\alpha$ interaction with other proteins in the developing cavefish lens.

In addition to functioning as a molecular chaperone during normal development and cellular stress, hsp90 appears to be involved in evolutionary processes such as the generation of novel phenotypes (Rutherford and Lindquist, 1998; Queitsch etal., 2002; Sollars et al., 2003). We have shown here that hsp90 $\alpha$ has an important role in regressive evolution of the eye in blind cavefish.

\section{Materials and Methods}

\section{Animals}

Astyanax surface fish were collected at Balmorhea State Park, Texas and cavefish were collected from La Cueva de El Pachón (Pachón cavefish) in Tamaulipas, Mexico and from La Cueva Chica (Chica cavefish) and El Sótano de la Tinaja (Tinaja cavefish) in San Luis Potosí, Mexico. Laboratory raised stocks were maintained in a custom built recycling 
aquarium system (Marine Biotechnology Inc, Beverly, MA). Embryos were obtained by natural spawning and cultured in recycled tank water (RTW) at $25^{\circ} \mathrm{C}$ as described previously (Jeffery et al., 2000). Under these conditions, gastrulation occurs at about 4-6 hours postfertilization (hpf), the tailbud stage at $10-11 \mathrm{hpf}$, the 18 somite stage at $16 \mathrm{hpf}$, lens vesicle formation at $22 \mathrm{hpf}$, hatching at $24 \mathrm{hpf}$, and lens apoptosis (in cavefish) begins at $36 \mathrm{hpf}$.

\section{Isolation of hsp90 $\alpha$, hsp90 $\beta$ and hsp70 cDNA}

Total RNA extracted from 18 somite surface fish was used to generate a Uni-ZAP XR cDNA library (Stratagene, La Jolla, CA). RT PCR was performed using the PCR Master Kit (Roche, Indianapolis, IN), cDNA library template, and the following degenerate oligonucleotide primers: hsp900 5'-TBMGRGARCTSATCTCCAAC-3' (forward) and 5'-RGWGATGTCATCDGGGTTHC-3' (reverse); hsp90ß 5'-TGATTGGGCAGTTTGGTGT-3' (forward) and 5'-GTCGTTGGTCAGGCTYTTGT-3' (reverse); hsp705'-ACGCAGCHAAGAACCAGGTG-3' (forward) and 5'-CGTGGGYTCGTTGATRAT-3' (reverse). After 35 PCR cycles at an annealing temperature of $55^{\circ} \mathrm{C}$, the reactions yielded fragments of the expected sizes: 516 bp ( $h s p 90 \alpha$ ), 580 bp ( $h s p 90 \beta$ ), and 375 bp ( $h s p 70$. These fragments were gel-extracted, ligated into the PCRScript vector (Stratagene), and sequenced. Blast analyses (Altschul et al., 1997) of all three nucleotide and translated amino acid sequences revealed significant and unmatched homology to corresponding zebrafish DNA fragments.

The full-length $h s p 90 \alpha$ coding sequence was obtained by screening the cDNA library with a digoxigenin-labeled DNA probe generated from the 516 bp PCR fragment of Astyanax Hsp90a. Two rounds of screening were conducted using Stratagene supplied procedures. After in vivo excision of the pBluescript SK phagemid (Stratagene), DNA sequence analysis of seven putative $H s p 90 \alpha$ clones revealed six containing the same complete 2170 bp coding region, which has been deposited in GenBank (AY222612).

\section{In situ hybridization}

The DIG RNA Labeling Kit (Roche) was used to generate antisense and sense digoxigenin-UTP-labeled RNA probes from the hsp90x, hsp90ß, and $h s p 70$ cloned fragments. At the appropriate stage, embryos were fixed in $4 \%$ paraformaldehyde (PFA)/PBS at $4^{\circ} \mathrm{C}$ overnight. They were then dehydrated in methanol and stored at $-20^{\circ} \mathrm{C}$. Prior to in situ hybridization, the fixed embryos were rehydrated in PBST (1X PBS, $0.1 \%$ Tween 20), hybridized at $65^{\circ} \mathrm{C}$ overnight, and stained following the procedures described by Püschel et al. (1992). After staining, embryos were photographed, embedded in polyester wax, and sectioned at $8 \mu \mathrm{m}$.

\section{Heat shock}

At $36 \mathrm{hpf}, 20-25$ surface fish or cavefish embryos were transferred to 60 $\mathrm{mm} \times 15 \mathrm{~mm}$ plastic petri dishes containing RTW, and the dishes were floated on the surface of a $37^{\circ} \mathrm{C}$ water bath. After $1 \mathrm{hr}$ at $37^{\circ} \mathrm{C}$, the heat shocked embryos, as well as $25^{\circ} \mathrm{C}$ control embryos from the same spawn, were processed for in situhybridization.

\section{TUNEL assay}

Fixed and dehydrated embryos were rehydrated in PBT (1x PBS, $0.1 \%$ Triton X-100). Embryos were then washed twice for 5 min with PBT, treated with Proteinase $\mathrm{K}(10 \mu \mathrm{g} / \mathrm{ml}$ in PBT) for $45 \mathrm{~min}$, washed twice for 5 min with PBT, post-fixed in 4\% PFA/PBT for $20 \mathrm{~min}$, and washed twice with PBT for $5 \mathrm{~min}$. These steps were carried out at room temperature. The TUNEL assay was performed using the In Situ Cell Death Detection Kit (Roche) with detection of fragmented DNA by peroxidase and Sigma Fast, 3,30diaminobenzidine (Sigma Chemicals, St. Louis, MO) according to instructions supplied by the manufacturers. Stained embryos were photographed, embedded in polyester wax, and sectioned at $8 \mu \mathrm{m}$.

\section{Pharmacological hsp90 inhibition}

At $24 \mathrm{hpf}$, groups of 25-30 cavefish embryos were transferred from RTW to $5 \mathrm{ml}$ of the following solutions: 10 or $20 \mu \mathrm{g} / \mathrm{ml}$ geldanamycin (Sigma) in
1\% DMSO/RTW, $2.5 \mu \mathrm{M}$ radicicol (Sigma) in 1\% DMSO/RTW, $1 \mu \mathrm{M}$ radicicol in 1\% DMSO/RTW, and 1\% DMSO/RTW (negative control). At 48 hpf all embryos treated with geldanamycin and $2.5 \mu \mathrm{M}$ radicicol, and half of the $1 \%$ DMSO control embryos, were fixed in $4 \%$ PFA/PBS and processed for in situhybridization or TUNEL. The $1 \mu \mathrm{M}$ radicicol treated and remaining $1 \%$ DMSO control embryos were allowed to continue development until 6 d post-fertilization (dpf), then were fixed in Bouin's solution overnight at $4^{\circ} \mathrm{C}$, dehydrated in ethanol, embedded in Paraplast, and sectioned at $8 \mu \mathrm{m}$. Sections were stained with eosin and hematoxylin following standard procedures.

\section{Acknowledgements}

This research was supported by NIH (EY014619) and NSF (IBN0110275) grants to W. R. J. We thank Allen Strickler for assistance in performing TUNEL assays.

\section{References}

ALTSCHUL, S. F., MADDEN, T. L., SCHAFFER, A. A., ZHANG, J., ZHANG, Z., MILLER, W., and LIPMAN, D. J. (1997). Gapped BLAST and PSI-BLAST: a new generation of protein database search programs. Nuc. Acids Res. 25:3389-3402.

BEEBE, J. C., and COATES, J. M. (2000). The lens organizes the anterior segment: specification of neural crest cell differentiation in the avian eye. Dev. Biol.220: 424431.

BEERE, H. M., WOLF, B. B., CAIN, K., MOSSER, D. D., MAHBOUBL, A., KUWANA, T., TAYLOR, P., MORIMOTO, R. I., COHEN, G., and GREEN, D. R. (2000). Heat shock protein 70 (HSP70) inhibits apoptosis by preventing recruitment of procaspase-9 to the Apaf-1 apoptosome. Nat. Cell Biol. 2: 469-475.

BLECHINGER. S. R., EVANS, T. G, TANG, P. T., KUWADA, J. Y., WARREN, J. T. JR, and KRONE, P. H. (2002). The heat-inducible zebrafish hsp70 gene is expressed during normal lens development under non-stress conditions. Mech. Dev. 112: 213-215.

CHEN, S., and SMITH, D. F. (1998). Hop as an adapter in the Hsp70/Hsp90 chaperone machinery. J. Biol. Chem. 272: 35194-35200.

CHOW, K-C. (2000). Hsp70 (DnaK)-an evolutionary facilitator? Trends Genet. 11: 484-485.

DASH, A, CHUNG, S., and ZELENKA, P. S. (1994). Expression of HSP70 mRNA in the embryonic chicken lens: association with differentiation. Exp. Eye Res. 58: 381-387.

DOWLING, T. E, MARTASIAN, D. P., and JEFFERY, W. R. (2002). Evidence for multiple genetic lineages with similar eyeless phenotypes in the blind cavefish, Astyanax mexicanus. Mol. Biol. Evol. 19: 446-455.

GUPTA, R. (1995). Phylogenetic analysis of the $90 \mathrm{kDa}$ heat shock gene family of protein sequences and an explanation of the relationship among animals, plants and fungi species. Mol. Biol. Evol. 12: 1063-1073.

JEFFERY, W. R. (2001). Cavefish as a model system in evolutionary developmental biology. Dev. Biol. 231: 1-12.

JEFFERY, W. R., AND MARTASIAN, D. P. (1998). Evolution of eye regression in the cavefish Astyanax. Apoptosis and the pax6gene. Am. Zool. 38: 685-696.

JEFFERY, W. R., STRICKLER, A. E., GUINEY, S., HEYSER, D. G., and TOMAREV, S. I., (2000). Prox 1 in eye degeneration and sensory organ compensation during development and evolution of the cavefish Astyanax. Dev. Genes Evol. 210: 223230.

JEFFERY, W. R., STRICKLER, A. E., and YAMAMOTO, Y. (2003). To see or not to see: evolution of eye degeneration in Mexican blind cavefish. Comp. Int. Biol. 43 : 435-446..

KRONE, P. H., and SASS, J. B. (1994). hsp90a and $h s p 90 \beta$ genes are present in zebrafish and are differentially regulated in developing embryos. Biochem. Biophys. Res. Commun. 204:746-752.

KURITA, R., SAGARA, H., AOKO, Y., LINK, B. A., ARAI, K., and WATANABE, S. (2003). Suppression of lens growth by $\alpha A$-crystallin promoter-driven expression of diptheria toxin results in disruption of retinal organization in zebrafish. Dev. Biol. 255: 113-127.

LANG, R. A. (1997). Apoptosis in mammalian eye development: Lens morphogenesis, vascular regression, and immune privilege. Cell Death \& Differ. 4: 12-20. 
LELE, Z., ENGEL, S., and KRONE, P. H. (1997). Hsp47and hsp70gene expression is differentially regulated in a stress- and tissue-specific manner in zebrafish embryos. Dev. Genet. 21: 123-133.

LELE, Z., HARTSON, S. D., CRISOFRE MARTIN, C., WHITESELL, L., MATTS, R. L., and KRONE, P. H. (1999). Disruption of zebrafish somite development in pharmacological inhibition of Hsp90. Dev. Biol. 210: 56-70.

LEWIS, J. DEVIN, A., MILLER, A., LIN, Y., RODRIGUEZ, Y., NECKERS, L., and LIU, Z-G. (2000). Disruption of Hsp90 function results in degradation of the death domain kinase, receptor-interacting protein (RIP), and blockage of tumor necrosis factor-induced nuclear factor-B activation. J. Biol. Chem. 275: 1051910526.

MORIMOTO, R. I., TISSIERES, A., and GEORGOPOULOS, C. (1994). The Biology of Heat Shock Proteins and Molecular Chaperones. Cold Spring Harbor Laboratory Press, Cold Spring Harbor, New York.

MOSSER, D. D., CARDON, A. W., BOURGET, L., MERIIN, A. B., SHERMAN, M. Y., MORIMOTO, R. I., and MASSIE, B. (2000). The chaperone function of hsp70 is required for protection against stress-induced apoptosis. Mol. Cell. Biol. 20: 7146-7159.

OGATA, Y., MIKI, T, and SEKIMIZU, K. (1993). Role of heat shock proteins for homologous recomibination in Escherichia coli. Biochem, Biophys. Res. Commun. 197: 34-39.

PANDEY, P., SALEH, A., NAKAZAWA, A., KUMAR, S., SRINIVASULA, S. M., KUMAR, V., WEICHSELBAUM, R., NALIN, C., ALNEMRI, E. S., KUFE, D., and KARBANDA, S. (2000). Negative regulation of cytochrome c-mediates oligomerization of Apaf- 1 and activation of procaspase- 9 by heat shock protein 90 . EMBO J. 19: 4310-4322.

PICARD, D., KHURSHEED, B., GARABEDIAN, M. J., FORTIN, M. G., LINDQUIST, S., and YAMAMOTO, K. R. (1990). Reduced levels of hsp90 compromise steroid receptor action in vivo. Nature 348: 166-168.

PÜSCHEL, A. W., GRUSS, P., and WESTERFIELD, M. (1992). Sequence and expression pattern of pax- 6 are highly conserved between zebrafish and mice. Development 114: 643-651.

QUEITSCH, C., SANGSTER, T. A., and LINDQUIST, S. (2002). Hsp90 as a capacitator of phenotypic variation. Nature 749: 1-7.

RICHTER, K., and BUCHNER, J. (2001). HSP90: chaperoning signal transduction. J. Cell. Physiol. 188: 281-290.

ROE, M. S., PRODROMOU, C., O'BRIEN, R., LADBURY, J. E., PIPER, P. W., and PEARL, L. H. (1999). Structural basis for inhibition of the hsp90 molecular chaperone by the antitumor antibiotics radicicol and geldanamycin. J. Med. Chem. 42: $260-266$.
RUTHERFORD, S. L., and LINDQUIST, S. (1998). Hsp90 as a capacitator for morphological evolution. Nature 396: 336-342.

SASS, J. B., WEINBERG, E. S., and KRONE, P. H. (1996). Specific localization of

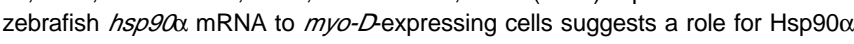
during normal muscle development. Mech. Dev. 54: 195-204.

SASS, J. B., MARTIN, C. C., and KRONE, P. H. (1999). Restricted expression of zebrafish hsp900 gene in slow and fast muscle fiber lineages. Int. J. Dev. Biol. 43: 835-838.

SMITH, D. F., STENAGARD, B. A., WELCH, W. J., and TOFT, D. O. (1992). Assembly of progesterone receptor with heat shock proteins and receptor activation are ATP mediated events. J. Biol. Chem. 267: 1350-1356.

SOLLARS, V., LU, X., XIAO, L., WANG, X., GARFINKEL, M. D., and RUDEN, D. (2003). Evidence for an epigenetic mechanism by which $\mathrm{Hsp} 90$ acts as a capacitor for morphological evolution. Nature Genet. 33: 70-74.

STRICKLER, A. G., FAMUDITIMI, K., and JEFFERY, W. R. (2002). Retinal homeobox genes and the role of cell proliferation in cavefish eye degeneration. Int. J. Dev. Biol. 46: $285-294$

VANDEN BERGHE, T., KALAI, M., VAN LOO, G., DECLERCQ, W., and VANDENABEELE, P. (2003). Disruption of HSP90 function reverts tumor necrosis factor-induced necrosis to apoptosis. J. Biol. Chem. 278: 5622-5629.

WHITSELL, L., MIMMNAUGH, E. G., DE COSTA, D., MYERS, C. E., and NECKERS L. M. (1994). Inhibition of heat shock protein HSP90-pp60v-src heteroprotein complex formation by benzquinone anasamycins: essential role of stress proteins in oncogenic transformation. Proc. Natl. Acad. Sci. USA. 91: 8324-8328.

WRIDE, M. A. (2000). Minireview. Apoptosis as seen through a lens. Apoptosis 3: 203209.

WRIDE, M. A., PARKER, E., and SANDERS, E. J. (1999). Members of the bcl-2 and caspase families regulate nuclear degeneration during chick lens fibre differentiation. Dev. Biol. 213, 142-156.

WU, Y., KITA, K., and SUZUKI, N. (2002). Involvement of human heat shock protein $90 \alpha$ in nicotine-induced apoptosis. Int. J. Cancer 100: 37-42.

XANTHOUDAKIS, S., and NICHOLSON, D. W. (2000). Heat-shock proteins as death determinants. Nature Cell Biol. 2: E163-E165.

YAMAMOTO, Y., and JEFFERY, W. R. (2000). Central role for the lens in cavefish eye degeneration. Science 289: 631-633.

XU, Y., and LINDQUIST, S. (1993). Heat shock protein hsp90 governs the activity of pp60v-src kinase. Proc. Nat. Acad. Sci. USA90: 7074-7078.

YOUNG, J. C., MOAREFI, I., and HARTL, F. U. (2001). Hsp90; a specialized but essential protein folding tool. J. Cell Biol. 154: 267-273. 